\title{
Electromagnetic Launcher : Review of Various Structures
}

\author{
Siddhi Santosh Reelkar \\ Department of Electrical Engineering, \\ Government College of Engineering, \\ Karad
}

\author{
Prof. Dr. U. V. Patil \\ Department of Electrical Engineering, \\ Government College of Engineering, \\ Karad
}

\author{
Prof. Dr. V. V. Khatavkar \\ Department of Electrical Engineering, \\ P.E.S. Modern college of Engineering, Pune
}

\author{
Hrishikesh Mehta \\ Aethertec Innovative Solutions, \\ Bavdhan, Pune
}

\author{
Utkarsh Alset \\ Aethertec Innovative Solutions, \\ Bavdhan, Pune
}

\begin{abstract}
A theoretic review of electromagnetic coil-gun launcher and its types are illustrated in this paper. In recent years conventional launchers like steam launchers, chemical launchers are replaced by electromagnetic launchers with auxiliary benefits. The electromagnetic launchers like rail- gun and coil-gun elevated with multi pole field structure delivers great muzzle velocity and huge repulse force in limited time. Various types of coil-gun electromagnetic launchers are compared in this paper for its structures and characteristics. The paper focuses on the basic formulae for calculating the values of inductance and resistance of electromagnetic launchers.
\end{abstract}

Keywords - Electromagnetic coil-gun launcher (EML), inductance, coil-gun types, multi pole field EML

\section{INTRODUCTION}

The first prototype of EML tested as aircraft in 1946, termed as Electropult, electromagnetic launcher replaced all traditional launcher with acquirement of more benefits viz eco-friendly, eliminates complex mechanism, reduce volume and weight, launch high weight objects, can be renewable, less maintenance, less risk, less manpower [1]. Since then, Electromagnetic launch (EML) is attracting researchers for development of launching technology.

EML system uses concept of linear electric motor to accelerate a projectile. This is used in wide-range military application, aircraft launching, antiterrorism weaponry, Electromagnetic mortars, Missile launcher to launch toxic waste into space, acceleration of objects with extremely high speed. There is a need to convert electric energy into kinetic energy, in this process huge amount of energy lost takes place in conversion process [2].

Electromagnetic have two basic categories, i.e. rail-gun launcher and coil-gun launcher [3]. Coil gun launcher is differentiated by its design structure and properties in five categories Induction coil-gun, Reconnection coil-gun, Reluctance coil-gun, helical coil-gun, Thompson's ring. EML consists of excitation and acceleration coils, projectile or object, capacitor charging circuit, capacitor bank, triggering switch.
This paper is mainly focusing the basic principle of electromagnetic coil-gun launcher, inductance and resistance calculations, construction and modeling concept of different coil-gun launcher.

\section{WORKING PRINCIPLE}

Rail gun has two parallel rails from which object is launched. When current passes through the rails to the object it produces arc. Because of high current pulse it has more contact friction losses [4]. Compare to the rail-gun launcher, Coil-gun launchers have no contact friction losses as there is no electrical contact between coils and object. The Electromagnetic coil-gun launcher consists of capacitor bank, solenoid coils and projecting objects [3]. The coil-gun launchers have series of solenoid coils which accelerate the projecting object placed inside the coils. These coils use electromagnet in the configuration of linear motor to accelerate a projecting object. The object and coils are arranged on common axis. Capacitor bank delivers large electric current to produce strong magnetic field essential for acceleration of projecting object $[3][5,6]$. The capacitor bank delivers high current pulse given to the excitation coil. The electric current energizes electromagnetic solenoid coils, electromagnetic force generated by Fleming's right hand rule. Magnitude of electromagnetic force experienced by object is represented by the Lorent' $z$ force given by [1]

$$
\begin{aligned}
& F=B I L \\
& \text { where, } \mathrm{B}=\text { magnetic field } \\
& \mathrm{I}=\text { current through armature } \\
& \mathrm{L}=\text { length of coil }
\end{aligned}
$$

The object has current from varying current in the solenoid coil which works as a primary excitation coil. There is a thrust force generated by the Lenz's Law which is based on Faraday's law [7-8].

$$
\varepsilon=N \frac{d \varphi}{d t}
$$

where, $\mathrm{N}=$ number of turns of coils

The magnitude of Emf induced in circuit is proportional to the rate of change of flux. The electromagnetic force of coils attracts and launches projectile the direction of induced 
current in magnetic field. The object is propelled by force generated in the direction of common axis through the accelerating coils around the object. As there is no mechanical friction, the velocity of projecting object is becoming very high [8]. To achieve maximum projectile velocity multiple coil design is suggested [5]. In multistage coil gun, the force creating process is repeated in every stage.

\section{INDUCTANCE AND RESISTANCE MEASUREMENT}

For computation of mutual inductance of coils traditional Fawzi / Burka's, Williamson / Leanard's, and Grover's numerical methods are used. To avoid singular cases in integration by using L'Hopital's Rule [9], new combined analytical and numerical method used for calculation of magnetic energy [7]. This method includes skin effect, so the current distribution is non-uniform in conductors. It divides cross section of conductors in mesh [9]. The final answer of this method gives closed loop function in terms of Jacobian Elliptical first and second order function and Heumann's Lambda function. This method is applicable to thin- wall solenoids, Disk coils, Two coaxial circular coils [7-9].

Inductance of non-coaxial coil is calculated by using Bessel function as it gives inductance as one dimensional integral of closed loop function [10]. There is only difference between co-axial and non-coaxial coil is a last term of Bessel function in non-coaxial coils. This method is applicable for cylindrical coil, thin-solenoid coil, pancake coil, circular coil

In multi-pole field electromagnetic launcher, inductance is calculated on the basis of position of projectile. ,

Inductance of coil is calculated when (a) projectile is completely inside the coil and (b) projectile is completely outside the coil. Let, $\mathrm{x}$ is a position of projectile [11-12].

$$
L(x)=\frac{N^{2}}{R_{\text {Total }}(x)}
$$

where, $\mathrm{R}_{\text {Total }}(\mathrm{x})=$ total reluctance of coil at $\mathrm{x}$ position of projectile.

When projectile is completely inside the coils then maximum and minimum value of inductance is achieved.

Reluctance of magnetic circuit is equivalent to the resistance of an electric circuit. As there is magnetic field produce by coils, so reluctance of coils required to calculate [11].

The basic formula for reluctance is

Reluctance $=$

$$
\text { length of magn }
$$

The reluctance in core includes relative permeability $\left(\mu_{\mathrm{r}}\right)$ and magnetic permeability of free space $\left(\mu_{0}\right)$. And reluctance in air gap include only magnetic permeability of free $\operatorname{space}\left(\mu_{0}\right)$. [13]

The resistance of excitation coil (catapult coil) is given by

$$
R=\frac{p l}{A}
$$

Inductance of catapult coil

$$
L_{e}=\frac{N^{2} A^{2}}{30 A-11 D_{i}}
$$

Where, Area of coil is calculated by

$$
A=D_{i}+N_{c} \frac{(w+s)}{2}
$$

Where, $\mathrm{D}_{\mathrm{i}}=$ inner diameter of catapult coil

$$
\begin{aligned}
& \mathrm{N}_{\mathrm{c}}=\text { no. of turns in catapult coil } \\
& \mathrm{w}=\text { wire diameter of catapult coil } \\
& \mathrm{s}=\text { distance between coil winding }
\end{aligned}
$$

Force generated on projectile is calculated from electromechanical energy conversion principle which use magnetic field as coupling medium between electrical and mechanical system. That shows electric energy produce by capacitor bank transfer into catapult coil which energies acceleration coils to create magnetic field. The projectile inside the acceleration coil experiences mechanical force given by, [14].

$$
F=I \int d l * B
$$

Force generated on projectile is

$F=\frac{i^{2}}{2} \frac{d L(x)}{d x}$

Force depends on mutual inductance and magnetic diffusion process in coil.

\section{CATEGORIES OF COIL-GUN LAUNCHER}

Coil launcher can be categorized into 5-types (i) Induction coil-gun launcher, (ii) Helical coil-gun launcher, (iii) Reconnection coil-gun launcher, (iv) Reluctance coil-gun launcher and (v) Thompson's Ring.

(i) Induction coil-gun launcher: Induction coil gun launchers have high conversion efficiency. When coils were fired, eddy current induced in projectile which produces repulsive accelerating force. To produce a greater force at beginning, at the bottom of coil pancake coil added [4]. It accelerates heavy projectile to much higher velocity.

Induction coil gun launchers are basically differentiated as [15]. (a) Asynchronous: The driving coils may be connected in series or in parallel and energized with a certain time sequence. The relative movement between the projectile and the travelling wave induces current in the projectile. (b) Synchronous: The driving coils are stacked end to end and energized sequentially to provide an impulse force to accelerate the projectile. The velocity of the projectile is exactly equal to that of the electromagnetic travelling wave [16].

(ii) Helical coil-gun launcher:

This involves separate drive coil and launch coil. It launches both hollow and solid projectiles. it operates at lower current and higher efficiency compared to induction coil-gun launcher. The driving coil is fixed on shield material like air shield, conductive shield, and ferrite shield [17].

(iii) Reconnection coil-gun launcher:

This coil-gun type had a shape like Disc. Acceleration coil is made of two coaxial copper coils and projectile is plate shape locate in gap between two accelerating coils. External coil produces time varying magnetic field which induces current in a conducting plate. A current is pulsed through the coil and produces eddy current which propel the projectile.

(iv) Reluctance coil-gun launcher:

It works on principle of reluctance motor [18], uses attractive ferromagnetic properties to accelerate the projectile. Coil is magnetized by carefully timing the 
coil current and the Attractive forces of coils pull the projectile upwards sequentially [5]. Its energy conversion efficiency is higher than the inductance coilgun because electrical energy conversion only takes place at excitation coil, so there is negligible eddy current in the projectile [19]. Timing the current pulse is crucial parameter of this launcher otherwise it can experience suck-back effect [5].

(v) Thompson's Ring: It is a higher voltage model of Induction coil gun. Initially spiral coil charged through capacitors then Thompson's ring creates its own magnetic field which is adversely to magnetic field of coil. the repulsion between the magnetic field accelerates ring upward.

\section{Multiple Field Electromagnetic Launcher}

To propel object or projectile in space with extremely high speed and in limited time it requires huge repulse force and long durative time simultaneously. To achieve this, multi pole field electromagnetic launchers are used [4]. Multi pole field electromagnetic launcher have Big caliber and multistage structure in which multi pole acceleration coils in one stage charged simultaneously by capacitor bank [20]. The acceleration coil with pulse current inducts the projectile to generate eddy current.

Authors [21], proposed multistage twisty multi pole electromagnetic launcher structure in which the second stage of coil is twisted by some angle to first stage for better performance. It results as accelerated speed and magnetic torque of rotational motion is larger and transverse displacement of projectile is less.

If the system is excited by sinusoidal current flowing in saddle coil moving in axial directions. Each driving coil produces magnetic field which direction along radius of cylinder. Thus, radial magnetic flux density with loop Eddy current produces large axial thrust force, and then radial compressive force decreases [22-23].

Radial magnetic field can be generated by increase in no. of poles, but complexity also increases. The connection pattern of driving coil should be like it can generate magnetic field in both inward and outward direction simultaneously to get magnetic lines reconnection.

Author [24], proposes toroidal field electromagnetic launcher with new accelerator mode to avoids catapult coil. It utilizes toroidal magnetic field interacting with radial current to generate axial accelerating force which avoids catapult coil for initial launch speed. Toroidal magnetic field interact with radial eddy current by axial acceleration force. Without catapult coil launcher has great axial acceleration force. Double armature multi pole field EML having the armature are placed inside as well as outside of driving coil [25] to improve efficiency but muzzle velocity is similar to single armature launch.

\section{DISCUSSION}

Based on results obtained and discussed by authors [1-31], following remarks major results can be shared,

a) As the number of poles in the multi-pole field acceleration coil is increased, the velocity and force applied on the projectile increases. b) Design of coil gun depends on magnetic flux density in air gap, maximum current density in coil cross section area and repulsive force acting on the coil.

c) With rated power supplies, air cored system can operate with greater magnetic field strength than iron cored system. so higher acceleration and force is achieved.

d) Increase a launching projectile speed and improve efficiency of coil gun, it can be possible by changing projectile length or width.

e) Reduction in gap between projectile and inner diameter of driving coil, increases speed of projectile.

f) Overall efficiency of induction coil-gun launcher is reduces as the magnetic coupling between the driving coil and projectile is become weak because of multilayer coils.

g) The maximum speed of projectile increases as the armature height longer than the driving coil height.

h) To achieve high muzzle velocity and better electromagnetic shielding effects, high permeability silicon steel sheet can be used as shield in helical coilgun launcher.

i) A large mutual inductance gradient and small system resistance is fruitful to achieve high efficiency of Helical coil-gun Electromagnetic launcher as a reliable and efficient medium-velocity launcher.

j) Multi pole field electromagnetic launchers uses multi pole magnetic field magnet or multistage magnet field interact with eddy current to accelerate the projectile to produce a huge propulsion force to accelerate projectile. Coils framed by superconducting wire to improve the running current and reduce the energy loss.

k) Multi pole field electromagnetic launcher design can improve the magnetic stability of the projectile and conserves high efficiency parameter of launcher.

1) Multipole field electromagnetic launcher has great axial component of axial acceleration force and high muzzle speed. And has potential of developing huge thrust force and super velocity launch.

m) Advantages of twisty M.F.E.L. are large driven mass, reliable spinning stability, suspension, Restoring force, high speed launch.

n) The current direction should be opposite in adjacent driving coil to achieve increase in overall efficiency with increase in initial voltage of capacitors. This type of coil connection pattern gives more uniform overall eddy current distribution and strong magnetic field.

o) Distance of projectile from coil in first stage is important factor affecting acceleration forces, so discharge time is basic requirement of coils design.

\section{CONCLUSION}

Collective results are briefly elaborated and concluding remarks are shared in this paper as a part of discussion. This will surely help to reduce the efforts of the researchers to bridge the gap in the research area, attract and further improve the performance of EML towards its effective practical and professional utilization.

\section{REFERENCES}

[1] Azeem Singh Kahlon, Taavishe Gupta, Pooja Dahiya, Sudhir Kumar Chaturvedi," A Brief Review on Electromagnetic Aircraft Launch 
System", International Journal of Mechanical and Production Engineering, Volume- 5, Issue-6, Jun.-2017.

[2] Daehwan Kwak, Yun Sik Jin, Young Bae Kim, Jong Soo Kim, Chuhyun Cho," Effects of Armature Height and Position on the Performance of Induction Coil Launcher", IEEE TRANSACTIONS ON PLASMA SCIENCE, July 8, 2017.

[3] Dong Yang, Zhenxiang Liu, Ting Shu, Lijia Yang," Design and testing of a coil-unit barrel for helical coil electromagnetic launcher", American Institute of Physics

[4] Yingwei Zhu, Yu Wang, Zhongming Yan, Liang Dong, Xiaofang Xie, and Haitao Li, "Multipole Field Electromagnetic Launcher," IEEE TRANSACTIONS ON MAGNETICS, VOL. 46, NO. 7, JULY 2010

5] Prof. Yogesh Fatangde, Swapnil Biradar, Aniket Bahmne, Suraj Yadav, Ajay Yadav, "Electromagnetic Coil Gun Launcher System", IJIRSET, VOL. 8, ISSUE NO. 3, MARCH 2019

[6] Naga Akhila madireddy, Dr. O. Chandrasekhar, "Laboratory model of Electromagnetic Propulsion Based coil gun", Restorating Justice in the Russian federation, ISSN 1943-023X, APRIL 2018

[7] Slobodan Babic and Cevdet Akyel," Improvement In Calculation of Self and Mutual Inductance of Thin Wall Solenoid and Disk Coils", IEEE TRANSACTIONS ON MAGNETICS, VOL.36, NO.4, JULY2000

[8] Su Jeong Lee, Jin Ho Kim, Soo-Hyun Kim, "Design and exeperiments of multi-stage coil gun system ", JOURNAL OF VIBROENGINEERING, VOL. 18, ISSUE 4, JUNE 2016

[9] Slobodan Babic and Cevdet Akyel ,New Analytic- Numerical Solutions For The Mutual Inductance Of Two Coaxial Circular Coils With Rectangular Cross Section In Air", IEEE TRANSACTIONS ON MAGNETICS, VOL.42,NO.6,JUNE2006

[10] John T. Conway, "Inductance Calculations For Non Coaxial Coils Using Bessel Functions", IEEE TRANSACTIONS ON MAGNETICS, VOL.43,NO.3,MARCH 2007

[11] K. Shrichandan, P. Mallikarjuna Rao, "Computation Of Magnetic Forces In Multipole Field Electromagnetic Launcher",ijmems, vol.4 no. 3,761-774, 2019

[12] K. Shrichandan, P. Mallikarjuna Rao, "A Mathematical Formulation Of Inductance For Multi Pole Field Electromagnetic Launcher", international journal of pure and apllied mathematics, vol. 118, no.24, 2018

[13] C.D.Sijoy, Shashank Chaturvedi, " Calculation Of Accurate Resistance And Inductance For Complex Magnetic Coils Using The FiniteDifference Time- Domain Technique For Electromagnetic", IEEE TRANSACTION ON PLASMA SCIENCE, VOL.36,NO.1, FEBRUARY 2008

[14] K.Sri Chandan, Dr. P. Mallikarjuna Rao," Comprehensive Analysis Of Octapole Electromagnetic Launcher",IJRASET, VOL.5, ISSUE X, OCTOBER 2017

[15] Ahmadali khatibzadeh, M.R. Besmi, "Improve Dimension Of Projectile For Increasing Efficiency Of Electromagnetic Launcher", IEEEPEDST, FEBRUARY 2013

[16] Tao Zhang, Wei Guo, Zizhou Su, Bin Cao, Ren Ren, Mingtao Li, Xia $\mathrm{Ge}$, Juxiang $\mathrm{Li}$, "Design and Evaluation of the Driving Coil on Induction Coilgun", IEEE TRANSACTION ON PLASMA SCIENCE, 2015

[17] Zhenxiang Liu, Dong Yang, Yang Li-jia, Shen Zhi,Ouyang Jian-ming, "Transient Simulation of Shielding Helical Coilgun", IEEE , 978-14673-0305-7, 2012

[18] Ferhat Daldaban, Vekil Sari, "The Optimization Of A Projectile From A Three- Coil Reluctance Launcher", Turk j Elect \& Comp Sci., 24: 2771-2788, April 2016

[19] Bofeg Zhu, Junyong Lu, Jie Wang, shincheng Xiong, "A Compulsator Driven Reluctance Coilgun-type Electromagnetic Launcher", IEEE TRANSACTION ON PLASMA SCIENCE, 2017

[20] T. G. Engel, W.C. Nunnally, J.M. Neri, "High- Efficiency, MediumCaliber Helical Coil Electromagnetic Launcher", IEEE TRANSACTION ON MAGNETICS, VOL. 41, NO.11, NOVEMBER 2005

[21] Zhu Yingwei, Wang Yu, Chen Weirong, Yan Zhongming, Li Haitao, "Analysis And Evaluation Of Three Stage Twisty Octapole Field Electromagnetic Launcher", IEEE TRANSACTION ON PLASMA SCIENCE, VOL.40,NO.5, MAY 2012
[22] Antonino Musolino, Racco Rizzo, Ernesto, "Travelling Wave Multipole Field Electromagnetic Launcher: An SOVP Analytical Model", IEEE TRANSACTION ON PLASMA SCIENCE, VOL.41, NO.5, MAY2013

[23] Wenbo Luo, Yu Wang, Zhixing Gui, Zhongming Yan, Weirong Chen, "Connection Pattern Research And Experimental Realization Of Single Stage Multipole Field Electromagnetic Launcher", IEEETRANSACTION ON PLASMA SCIENCE, VOL.41, NO.11, NOVEMBER 2013

[24] Xinpeng Xue, Tao Shu,Zhiyong Yang, Gang Feng, "Toroidal Field Electromagnetic Launcher", IEEE TRANSACTION ON PLASMA SCIENCE, 2016

[25] Zhongming Yan, Xiaofei Long, Falong Lu, Yu Wang, Hunjun Liu, "Study Of Single-Stage Double -Armature Multipole Field Electromagnetic Launcher", IEEE TRANSACTION ON PLASMA SCIENCE, 2017

[26] Ahmadali khatibzadeh, M.R. Besmi, "Improve Dimension Of Projectile For Increasing Efficiency Of Electromagnetic Launcher”, IEEEPEDST, FEBRUARY 2013

[27] Dr. Harry D. Fair," Electromagnetic Launch", International Journal of Impact Engineering 29(2003) 247-262, 2003

[28] Tao Zhang, Wei Guo, Fuchang Lin, Bin Cao, Zhiqiang Dong, Ren Ren, Kai Huang, and Zizhou Su," Experimental Results From a 4-Stage Synchronous Induction Coilgun", IEEE TRANSACTIONS ON PLASMA SCIENCE, VOL. 41, NO. 5, MAY 2013

[29] T. G. Engel, "Solid Projectile Helical coil Electromagnetic Launher", IEEE, 2007

[30] Liang Dong, Seliang Li,"Multipole Field Reconnection Electromagnetic Launcher", IEEE TRANSACTION ON PLASMA SCIENCE,VOL.46NO.2,FEB.2018

[31] Xinpeng Xue, Tao Shu,Zhiyong Yang, Gang Feng, "Toroidal Field Electromagnetic Launcher", IEEE TRANSACTION ON PLASMA SCIENCE,2016. 